\title{
Susceptibility of community Gram-negative urinary tract isolates to mecillinam and other oral agents
}

\author{
Tony Mazzulli MD FRCPC ${ }^{1}$, Martin Skulnick FIMLS ART ${ }^{1}$, Glen Small ART ${ }^{1}$, \\ Wayne Marshall ART ${ }^{1}$, Darryl J Hoban MD FRCPC ${ }^{2}$, George G Zhanel PharmD PhD ${ }^{2}$, \\ Susan Finn $\mathrm{ART}^{3}$, Donald E Low MD FRCPC ${ }^{1}$
}

\begin{abstract}
T Mazzulli, M Skulnick, G Small, et al. Susceptibility of community Gram-negative urinary tract isolates to mecillinam and other oral agents. Can J Infect Dis 2001;12(5):289-292.

OBJECTIVE: To determine the susceptibility of community outpatient Gram-negative urinary tract isolates to mecillinam and other commonly used oral agents.

DESIGN AND SETTING: The study was a laboratory-based study of consecutive Gram-negative urinary tract isolates. Only those isolates considered to be significant pathogens were included in the study. Susceptibility testing was performed using agar dilution methodology following guidelines published by the National Committee for Clinical Laboratory Standards. POPULATION STUDIED: Outpatients presenting to a family physician or clinic with signs or symptoms suggestive of a urinary tract infection were included in the study.

MAIN RESULTS: Of 2000 consecutive community isolates (91.8\% Escherichia coli, 3.9\% Klebsiella species, $2.0 \%$ Proteus species, $2.3 \%$ others), in vitro susceptibilities were: mecillinam $98.8 \%$, ampicillin $77.0 \%$, ciprofloxacin $100 \%$, trimethoprim/sulfamethoxazole $91.6 \%$ and nitrofurantoin $95.4 \%$. Susceptibility to mecillinam was significantly better than all other agents except ciprofloxacin $(\mathrm{P}<0.001$, McNemar's test). Organisms with reduced susceptibility to mecillinam included Citrobacter species, Pseudomonas aeruginosa and Providencia species.

CONCLUSIONS: Community Gram-negative urinary tract isolates remain highly sensitive to mecillinam and ciprofloxacin, but a significant number have developed resistance to trimethoprim/sulfamethoxazole. Further studies are required to determine the clinical significance of these results.
\end{abstract}

Key Words: Gram-negative organisms; Outpatients; Urinary tract infections

Pour le résumé, voir page suivante

The article was presented in part at the 38th Interscience Conference on Antimicrobial Agents and Chemotherapy, San Diego, California, USA, September 24 to 27, 1998 (Abst E-038)

${ }^{1}$ Department of Microbiology, Mount Sinai and Princess Margaret Hospitals, University of Toronto, Toronto, Ontario; ${ }^{2}$ Department of Clinical Microbiology, Health Sciences Centre, University of Manitoba, Winnipeg, Manitoba; ${ }^{3}$ Med-Chem Health Care Ltd, Toronto, Ontario Correspondence and reprints: Dr T Mazzulli, Mount Sinai Hospital, Department of Microbiology, Room 1485, 600 University Avenue, Toronto, Ontario M5G 1X5. Telephone 416-586-4695, fax 416-586-8746.e-mail tmazzulli@mtsinai.on.ca

Received for publication October 30, 2001. Accepted January 24, 2001 


\title{
La sensibilité des isolats des voies urinaires à Gram négatif au mécillinam et autres agents oraux dans la communauté
}

\begin{abstract}
OBJECTIF : Déterminer la sensibilité des isolats des voies urinaires à Gram négatif au mécillinam et autres agents oraux dans la communauté.

MODÈLE ET CONTEXTE : L'étude menée en laboratoire portait sur des isolats des voies urinaires à Gram négatif consécutifs. Seuls les isolats considérés comme des agents pathogènes importants ont été retenus dans l'étude. Les tests de sensibilité ont été menés en utilisant la technique de dilution en gélose, suivant les lignes directrices publiées par le National Committee for Clinical Laboratory Standards.

POPULATION ÉTUDIÉE : L'étude a été menée auprès de malades externes consultant un médecin de famille ou une clinique et manifestant des signes ou symptômes d'infection des voies urinaires.

PRINCIPAUX RÉSULTATS : Sur 2000 isolats de malades externes consécutifs (91,8\% d'Escherichia Coli, 3,9\% du genre Klebsiella, 2,0 \% du genre Proteus, autres 2,3\%), les sensibilités in vitro ont été de : mécillinam, 98,8 \% ; ampicilline, 77,0 $\%$; ciprofloxacine, $100 \%$; triméthoprime-sulfaméthoxazole, $91,6 \%$; et nitrofurantoïne, 95,4 \%. La sensibilité au mécillinam était significativement meilleure qu'à tous les autres agents sauf la ciprofloxacine $(\mathrm{P}<0,001$, test de McNemar). Les organismes moins sensibles au mécillinam étaient les genres Citrobacter, Pseudomonas aeruginosa et Providencia.

CONCLUSIONS : Les isolats des voies urinaires à Gram négatif dans la communauté demeurent extrêmement sensibles au mécillinam et à la ciprofloxacine, mais bon nombre ont acquis une résistance au triméthoprime-sulfaméthoxazole. Des études plus approfondies sont requises pour déterminer la signification clinique de ces résultats.
\end{abstract}

A cute urinary tract infections (UTIS) remain one of the most common problems for which patients seek medical treatment in the community. An estimated $10 \%$ to $20 \%$ of women will experience at least one infection during their lifetime (1). Fortunately, most episodes of uncomplicated UTIS do not result in long term sequelae. Appropriate antimicrobial treatment for UTIs increases the probability of suppression and cure $(2,3)$.

More than $90 \%$ of UTIs are due to enteric Gram-negative organisms, of which more than $80 \%$ are Escherichia coli $(4,5)$. Current management guidelines recommend empirical therapy for acute, uncomplicated lower UTIs in young women $(2,3)$. In typical cases, a urine culture is not necessary. Although this approach to management is simple and relatively cost effective, it is dependent on having an antimicrobial agent that is safe and to which most causative organisms are susceptible. Recent guidelines from the Infectious Disease Society of America (6) have recommended three days of treatment with trimethoprim/sulfamethoxazole or trimethoprim alone for the empirical therapy of acute uncomplicated cystitis. In areas where the prevalence of resistance to these agents is more than $10 \%$ to $20 \%$, the guidelines recommend the use of a fluoroquinolone. This is supported by a recent study by Talan et al (7), which showed that in patients with acute uncomplicated pyelonephritis, resistance to trimethoprim/sulfamethoxazole was associated with a significantly greater bacteriological and clinical failure rate. However, reports of increasing resistance of Gram-negative bacteria to trimethoprim/sulfamethoxazole, trimethoprim and amoxicillin, as well as concern about the potential for increasing resistance to fluoroquinolones with increasing use, emphasize the need for alternative agents $(2,8)$. Mecillinam is a novel oral beta-lactam antibiotic with considerable in vitro activity against the Enterobacteriaceae family $(5,9)$. Although this agent has been available for years, few clinical trials have evaluated its efficacy or in vitro activity against community Gram-negative uropathogens $(5,10-12)$. Thus, the purpose of this study was to determine the susceptibility of community outpatient

Gram-negative urinary tract isolates to mecillinam and compare this with other commonly used oral agents for the treatment of acute uncomplicated cystitis.

\section{MATERIALS AND METHODS}

Between July and December 1997, 2000 consecutive community Gram-negative urinary tract isolates were collected from patients presenting to family physicians offices or outpatient clinics in southern Ontario. Urine specimens were collected for culture and sensitivity testing at the discretion of the physician, and not solely for the purpose of this study. Specimens were initially processed by a private laboratory servicing outpatient offices and clinics. Only those isolates that were deemed to be significant urinary pathogens by standard laboratory criteria were included (13) and were subsequently sent to the Department of Microbiology at Mount Sinai Hospital (Toronto, Ontario) for susceptibility testing for the study. All isolates were identified using standard laboratory methods. Susceptibility testing against mecillinam, ampicillin, trimethoprim/sulfamethoxazole, nitrofurantoin and ciprofloxacin was performed using agar dilution methodology. The breakpoints for ampicillin, trimethoprim/sulfamethoxazole, nitrofurantoin and ciprofloxacin were those published by the National Committee for Clinical Laboratory Standards approved document M7-A4 (14). The mecillinam susceptibility and resistance breakpoints used were less than $16 \mathrm{mg} / \mathrm{mL}$ and $16 \mathrm{mg} / \mathrm{mL}$ or greater, respectively. $E$ coli ATCC 25922 and Pseudomonas aeruginosa ATCC 27853 were used as control strains, as recommended by the National Committee for Clinical Laboratory Standards (14). The susceptibility of mecillinam was compared with the susceptibility of the other agents using McNemar's test, with exact calculation of the test probability. Exact $95 \%$ CIs for percentages were calculated by using the F distribution $(15,16)$.

\section{RESULTS}

Of the 2000 consecutive community Gram-negative isolates, $1832(91.8 \%)$ were $E$ coli, 78 (3.9\%) were Klebsiella 
species, 41 (2.0\%) were Proteus species, and the remainder (2.3\%) were other Gram-negative organisms. Table 1 illustrates the activity of the different antibiotics against all 2000 urinary tract isolates. Mecillinam's activity was significantly better $(\mathrm{P}<0.001$, McNemar's test) than that of ampicillin, trimethoprim/sulfamethoxazole and nitrofurantoin but was inferior to ciprofloxacin. Table 2 shows the susceptibility of the various antimicrobial agents by organism. Against $E$ coli, mecillinam had significantly greater activity than trimethoprim/sulfamethoxazole and ampicillin, and had comparable activity with nitrofurantoin and ciprofloxacin. Mecillinam maintained significant activity against organisms that were either susceptible or resistant to the other antimicrobial agents tested (Table 3).

\section{DISCUSSION}

Our results suggest that most community Gram-negative urinary tract isolates remain susceptible to mecillinam and that this agent can be used for the empirical therapy of acute uncomplicated lower UTIs in the community. It has significantly better activity than all other agents tested except for ciprofloxacin. Compared with ampicillin, a commonly used beta-lactam antibiotic for the treatment of UTIs in pregnant women (17), mecillinam had significantly better activity against both ampicillin-sensitive and ampicillin-resistant isolates. This likely reflects the fact that mecillinam preferentially binds to penicillin-binding protein 2 in Gram-negative isolates, whereas other beta-lactams preferentially bind to penicillinbinding proteins $1 \mathrm{a}, 1 \mathrm{~b}$ or $3(18)$. As well, mecillinam has been shown to resist hydrolysis by beta-lactamases and thus has greater antibacterial potency against the Enterobacteriaceae family than other beta-lactam antibiotics (19). The relatively high rate of in vitro activity of mecillinam was also demonstrated for both susceptible and resistant isolates to trimethoprim/sulfamethoxazole and nitrofurantoin. The increased rates of resistance to trimethoprim/sulfamethoxazole may reflect the fact that it is one of the most commonly prescribed antibacterial agents in Canada for the treatment of uncomplicated UTIs, whereas mecillinam has not been used in Canada (20).

In a recent study of 2000 outpatient urinary tract isolates collected from tertiary care hospitals from across Canada, Zhanel et al (12) reported resistance rates for $E$ coli that were significantly higher than those found in our study for ampi-
TABLE 1

Activity of antibiotics against 2000 Gram-negative urinary tract isolates

\begin{tabular}{lcc}
\hline & $\begin{array}{c}\text { Percentage of } \\
\text { susceptible } \\
\text { isolates } \mathbf{( 9 5 \% ~ C l )}\end{array}$ & $\begin{array}{c}\text { Comparison with } \\
\text { activity of mecillinam } \\
\text { (McNemar's Test) }\end{array}$ \\
\hline Mecillinam & $98.8(98.2$ to 99.2$)$ & \\
Ampicillin & $77.0(75.0$ to 78.8$)$ & $\mathrm{P}<0.001$ \\
$\begin{array}{l}\text { Ciprofloxacin } \\
\text { Trimethoprim/ }\end{array}$ & $100.0(99.7$ to 100.0$)$ & $\mathrm{P}<0.001$ \\
$\quad$ sulfamethoxazole & $91.6(90.3$ to 92.8$)$ & $\mathrm{P}<0.001$ \\
Nitrofurantoin & $95.4(94.3$ to 96.2$)$ & $\mathrm{P}<0.001$ \\
\hline
\end{tabular}

cillin (41\% versus $18 \%$ ), mecillinam ( $7.4 \%$ versus $0.3 \%$ ) and trimethoprim/sulfamethoxazole (18.9\% versus $9.2 \%)$. Similar differences were noted among other Gram-negative isolates. These higher rates of resistance reported by Zhanel et al likely reflect the different patient populations studied. Although both studies were completed with outpatients, those patients in the study by Zhanel et al (12) presented to a tertiary care hospital, whereas those in our study were patients seen in family physician offices and outpatient clinics. Patients presenting to a tertiary care hospital may have more complicated UTIs, may have recently been inpatients and, thus, exposed to more resistant hospital flora, or may have failed previous therapy, all of which may account for the increased resistance compared with patients with simple uncomplicated UTIs presenting to their family physician. Because clinical data were not collected in this study or reported in the study by Zhanel et al (12), we could not determine any differences between the two patient populations to account for the differences in resistance rates. A similar trend toward higher resistance rates among outpatient urinary tract isolates received at a university microbiology laboratory and those from general practice has been noted previously (10). The university laboratory reported resistance rates that were three to four times higher for mecillinam and nitrofurantoin than those in general practice. Together with our results, this suggests that one must carefully interpret reported rates of resistance when selecting an antimicrobial agent for therapy of UTIs in the community. Reports from tertiary care laboratories may overestimate resistance rates in the community, even though the population studied may be outpatients.

TABLE 2

In vitro susceptibility of community Gram-negative uropathogens to selected antimicrobial agents

\begin{tabular}{|c|c|c|c|c|c|c|}
\hline \multirow[b]{2}{*}{ Organism } & \multicolumn{6}{|c|}{ Percentage of susceptible organisms by antibiotic (n) } \\
\hline & n (\%) & Ampicillin & Trimethoprim/sulfamethoxazole & Nitrofurantoin & Ciprofloxacin & Mecillinam \\
\hline Escherichia coli & $1832(91.8)$ & $82.0(1502)$ & $91.8(1681)$ & $99.5(1822)$ & $100(1832)$ & $99.7(1827)$ \\
\hline Klebsiella spcies & 78 (3.9) & $0.0(0)$ & $97.4(76)$ & $69.2(54)$ & $98.7(77)$ & $100(78)$ \\
\hline Enterobacter species & $19(0.9)$ & $0.0(0)$ & $100(19)$ & $63.0(12)$ & $100(19)$ & $100(19)$ \\
\hline Proteus species & $41(2.0)$ & $92.7(38)$ & $82.9(34)$ & $4.9(2)$ & $100(41)$ & $97.6(40)$ \\
\hline Citrobacter species & $14(0.7)$ & $0.0(0)$ & $100(14)$ & $100(14)$ & $100(14)$ & $92.8(13)$ \\
\hline Pseudomonas aeruginosa & $7(0.3)$ & $0.0(0)$ & $0.0(0)$ & $0.0(0)$ & $100(7)$ & $57.1(4)$ \\
\hline Providencia species & $5(0.2)$ & $0.0(0)$ & $100(5)$ & $0.0(0)$ & $100(5)$ & $0.0(0)$ \\
\hline Morganella morganii & $4(0.2)$ & $25(1)$ & $100(4)$ & $75(3)$ & $100(4)$ & $100(4)$ \\
\hline
\end{tabular}


TABLE 3

Activity of mecillinam against antibiotic-susceptible and resistant Gram-negative uropathogens

\begin{tabular}{|c|c|c|c|}
\hline & $\begin{array}{l}\text { Number of } \\
\text { isolates }\end{array}$ & $\begin{array}{l}\text { Number of } \\
\text { mecillinam- } \\
\text { susceptible } \\
\text { isolates }\end{array}$ & $\begin{array}{c}\text { Percentage of } \\
\text { mecillinam- } \\
\text { susceptible } \\
\text { isolates }(95 \% \mathrm{CI})\end{array}$ \\
\hline \multicolumn{4}{|l|}{ Ampicillin } \\
\hline Susceptible & 1539 & 1535 & 99.7 (99.3 to 99.9$)$ \\
\hline Resistant & 461 & 441 & 95.7 (93.4 to 97.3$)$ \\
\hline \multicolumn{4}{|l|}{ Ciprofloxacin } \\
\hline Susceptible & 1999 & 1975 & 98.8 (98.2 to 99.2$)$ \\
\hline Resistant & 1 & 1 & \\
\hline \multicolumn{4}{|c|}{$\begin{array}{l}\text { Trimethoprim/ } \\
\text { sulfamethoxazole }\end{array}$} \\
\hline Susceptible & 1832 & 1819 & 99.3 (99.8 to 99.6$)$ \\
\hline Resistant & 168 & 157 & 93.5 (88.6 to 96.7$)$ \\
\hline \multicolumn{4}{|l|}{ Nitrofurantoin* } \\
\hline Susceptible & 1889 & 1880 & 99.4 (99.1 to 99.8$)$ \\
\hline Resistant & 92 & 78 & 84.8 (75.8 to 91.4$)$ \\
\hline Total & 2000 & 1976 & 98.8 (98.2 to 99.2$)$ \\
\hline
\end{tabular}

*1981 isolates evaluable for this analysis

Resistance rates in our study were highest among organisms such as $P$ aeruginosa, Providencia species and Citrobacter species. Fortunately, these organisms are relatively uncommon causes of community-acquired lower UTIs, accounting for only $1.2 \%$ of all isolates in our study. The relatively high rates of susceptibility for most common organisms, such as $E$ coli, suggest that the empirical use of mecillinam for the treatment of uncomplicated outpatient UTIs would be successful. Our results are consistent with a previously reported Canadian study that showed that mecillinam had significantly better activity than ampicillin and trimethoprim/sulfamethoxazole against urinary tract Gram-negative isolates (21). In fact, mecillinam was active against $91.9 \%$ of ampicillin-resistant $E$ coli and $95.9 \%$ of trimethoprim/sulfamethoxazole-resistant $E$ coli. Although mecillinam has little in vitro activity against Gram-positive organisms and these were not included in our study, some studies have demonstrated the clinical efficacy of mecillinam in the treatment of uncomplicated lower UTIs due to organisms such as Staphylococcus saprophyticus $(22,23)$. Further clinical studies are required to determine whether the in vitro susceptibility of mecillinam translates into clinically effective therapy, especially in a three-day drug regimen as is currently recommended for trimethoprim/sulfamethoxazole and ciprofloxacin.

ACKNOWLEDGEMENTS: This study was supported in part by a grant from Leo Pharma Inc (Ajax, Ontario).

\section{REFERENCES}

1. Kunin CM. Detection, Prevention and Management of Urinary Tract Infections. Philadelphia: Lea \& Febiger, 1997.

2. Hooton TM, Stamm WE. Diagnosis and treatment of uncomplicated urinary tract infection. Infect Dis Clin North Am 1997;11:551-81.
3. Johnson JR. Urinary tract infections: molecular pathogenesis and clinical management. In: Mobley HLT, Warren JW, eds. Treatment and Prevention of Urinary Tract Infections. Washington, DC: American Society for Microbiology Press, 1996:95-118.

4. Stamm WE. Approach to the patient with urinary tract infection. In: Gorbach SL, Bartlet JG, Blacklow RR, eds. Infectious Diseases. Philadelphia: Saunders, 1992:788-98.

5. Zhanel GG, Karlowsky JA, Schwartz B, Jensen SB, Hoban DJ. Mecillinam activity compared to ampicillin, trimethoprim/sulfamethoxazole, ciprofloxacin and nitrofurantoin against urinary tract isolates of gram-negative bacilli. Chemotherapy 1998;44:391-6.

6. Warren JW, Abrutyn E, Hebel JR, Johnson JR, Schaeffer AJ, Stamm WE. Guidelines for antimicrobial treatment of uncomplicated acute bacterial cystitis and acute pyelonephritis in women. Clin Infect Dis 1999;29:745-58.

7. Talan DA, Stamm WE, Hooton TM, et al. Comparison of ciprofloxacin (7 days) and trimethoprim-sulfamethoxazole (14 days) for acute uncomplicated pyelonephritis in women: a randomized trial. JAMA 2000;283:1583-90.

8. Gupta K, Scholes D, Stamm WE. Increasing prevalence of antimicrobial resistance among uropathogens causing acute uncomplicated cystitis. JAMA 1999;281:736-8.

9. Neu HC. Synergy of mecillinam, a beta-amidinopenicillanic acid derivative, combined with beta-lactam antibiotics. Antimicrob Agents Chemother 1976;10:535-42.

10. Baerheim A, Digranes A, Hunskaar S. Are resistance patterns in uropathogens published by microbiological laboratories valid for general practice? APMIS 1999;107:676-80.

11. Pitkajarvi T, PyyKonen ML, Kannisto K, Piipo T, Viita P. Pivmecillinam treatment in acute cystitis. Three versus seven days study. Arzneimittelforschung 1990;40:1156-8.

12. Zhanel GG, Karlowsky JA, Harding GK, et al. A Canadian national surveillance study of urinary tract isolates from outpatients: comparison of the activities of trimethoprimsulfamethoxazole, ampicillin, mecillinam, nitrofurantoin, and ciprofloxacin. The Canadian Urinary Isolate Study Group. Antimicrob Agents Chemother 2000;44:1089-92.

13. Clarridge JE, Johnson JR, Pezzlo MT. Cumitech 2B, Laboratory Diagnosis of Urinary Tract Infections. Washington, DC: American Society for Microbiology, 1998.

14. National Committee for Clinical Laboratory Standards. Methods for dilution antimicrobial susceptibility tests for bacteria that grow aerobically. Approved standard M7-A4. Wayne: National Committee for Clinical Laboratory Standards, 1997.

15. Armitage P, Berry G. Statistical Methods in Medical Research, 2nd edn. Oxford: Blackwell, 1987:117-20.

16. Statistical Software for Exact Nonparametric Inference User Manual. Cambridge: Cytel Software Corporation, StatXact-Turbo for Sun, 1992.

17. Patterson TF, Andriole VT. Detection, significance and therapy of bacteriuria in pregnancy. Update in the managed health care era. Infect Dis Clin North Am 1997;11:593-608.

18. Spratt BG. The mechanism of action of mecillinam. J Antimicrob Chemother 1977;3:13-9.

19. Sougakoff $\mathrm{W}$, Jarlier V. Comparative potency of mecillinam and other $\beta$-lactam antibiotics against Escherichia coli strains producing different $\beta$-lactamases. J Antimicrob Chemother 2000;46(Suppl S1):9-14.

20. Naber KG. Survey of antibiotic usage in the treatment of urinary tract infections. J Antimicrob Chemother 2000;46(Suppl S1):49-52.

21. Zhanel GG, Karlowsky JA, Schwartz B, Jensen SB, Hoban DJ. Mecillinam activity compared to ampicillin, trimethoprim/sulfamethoxazole, ciprofloxacin and nitrofurantoin against urinary tract isolates of $\mathrm{G}$ ram-negative bacilli. Chemotherapy 1998;44:391-6.

22. Granlund M, Landgren E, Henning C. Pivmecillinam in treatment of Staphylococcus saprophyticus urinary tract infections. Scand J Infect Dis 1983;15:65-9.

23. Hovelius B, Mardh PA, Nygaard-Pedersen L, Wathne B. Nalidixic acid and pivmecillinam for treatment of acute lower urinary tract infections. Scand J Prim Health Care 1985;3:227-32. 


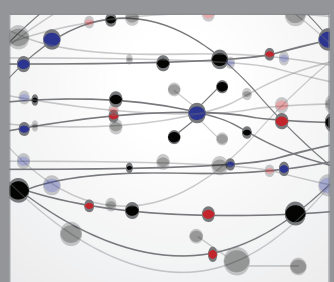

The Scientific World Journal
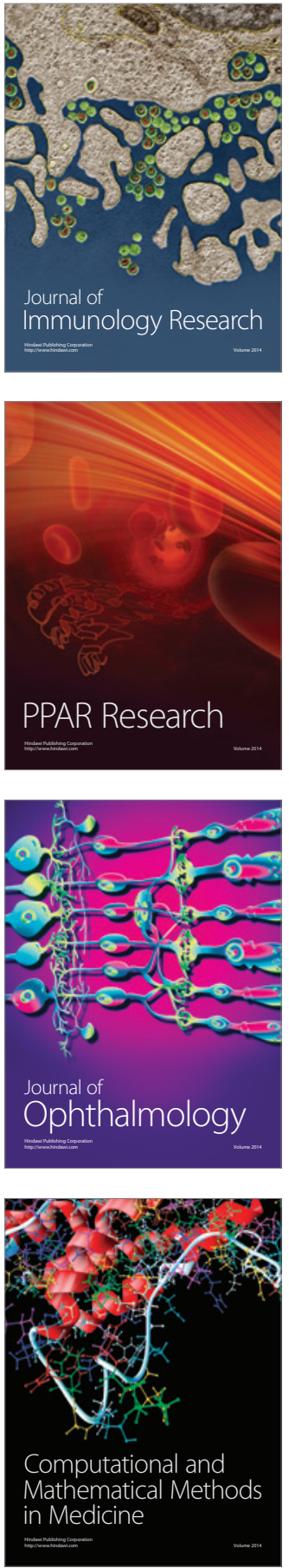

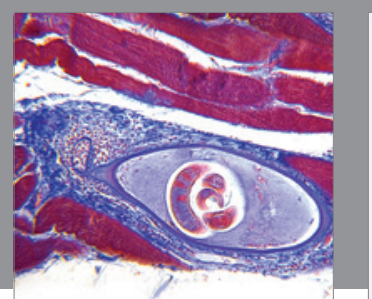

Gastroenterology Research and Practice

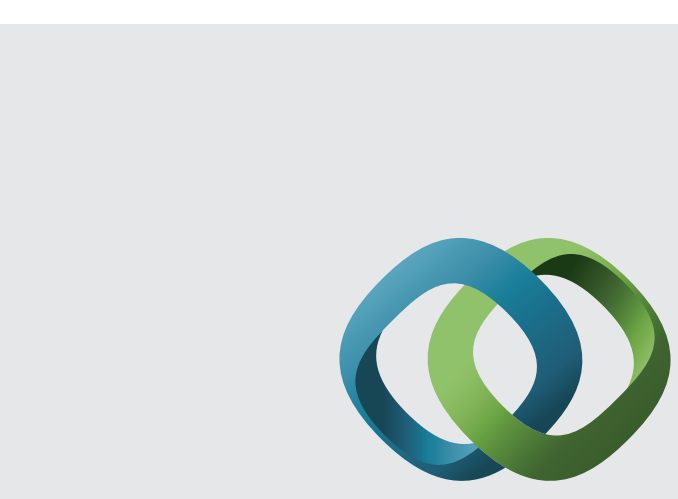

\section{Hindawi}

Submit your manuscripts at

http://www.hindawi.com
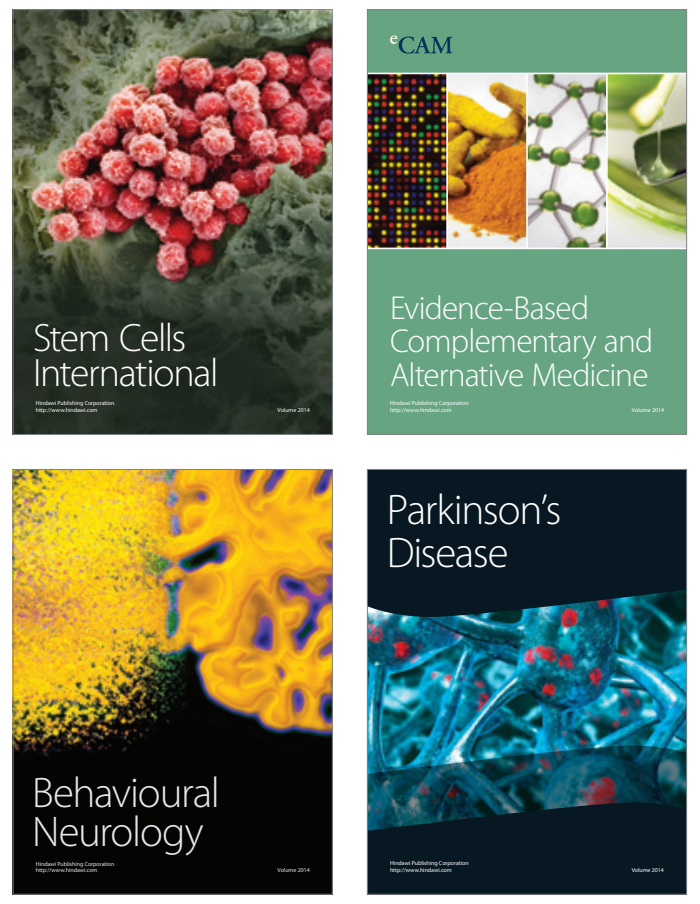
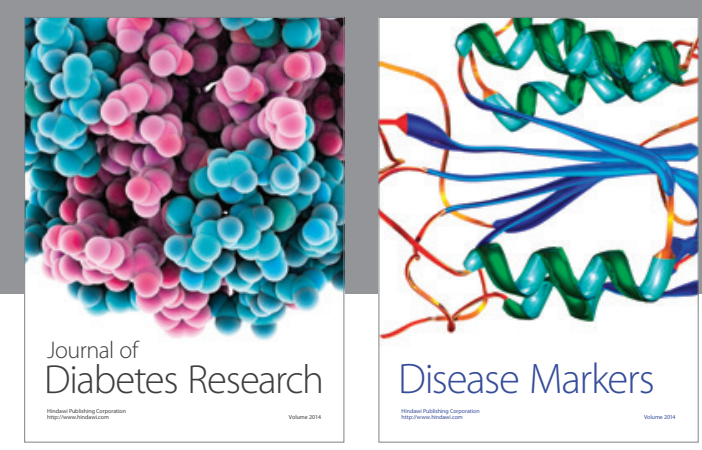

Disease Markers
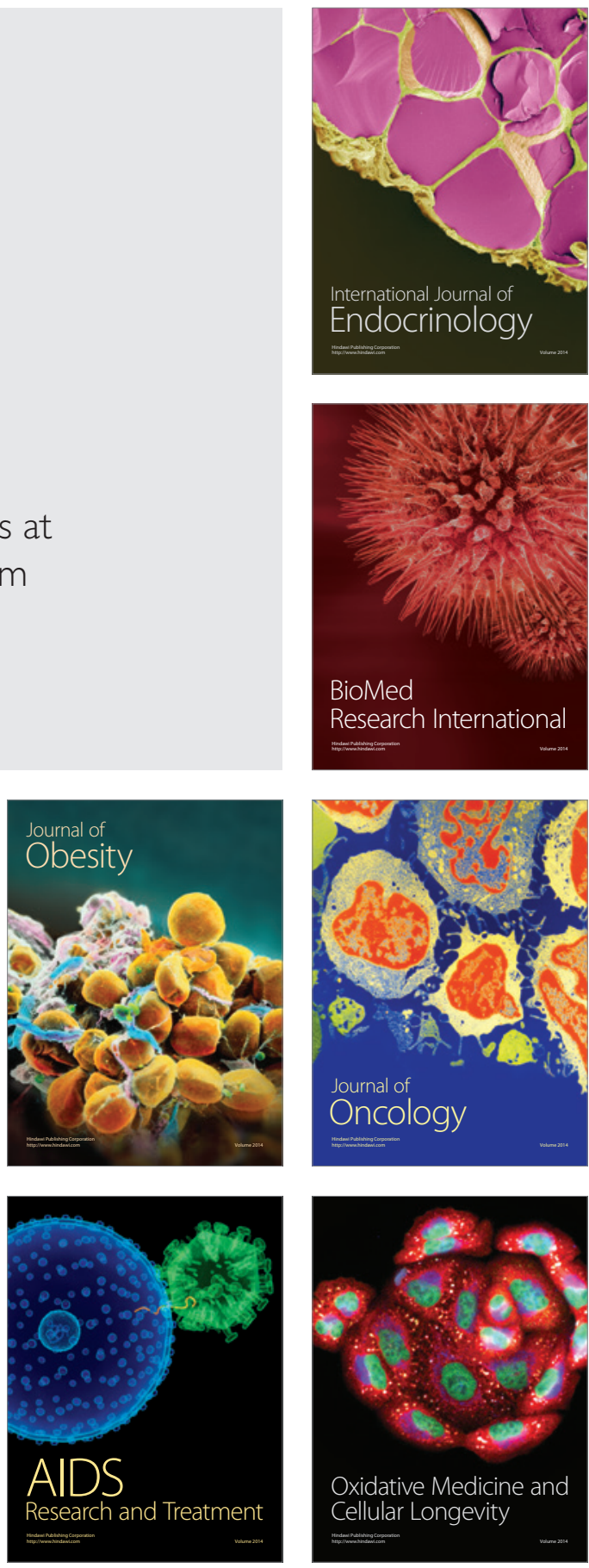